Entgeltliche Einschaltung

Fachkurzinformation siehe Seite 28

(c) Springer Medizin Wien

Quelle: Therapie-Management in der SpA: Interdisziplinäre Zusammenarbeit zum Vorteil der Patienten. Satellitensymposium von AbbVie im Rahmen der Jahrestagung der Österreichischen Gesellschaft für Rheumatologie und Rehabilitation, 28.11.2013, Wien

Referenzen:

1. Rudwaleit M et al.: Ann Rheum Dis 2009; 68:777-783

2. Boonen $A$, van der Linden SM: J Rheumatol Suppl. 2006;78:4-11. Review.

3. Smolen J et al.: Ann Rheum Dis. 2014;73(1):6-16

4. van der Heijde DM et al.: Arthritis Res Ther. 2009;11(4):R124

5. Baraliakos $X$ et al.: Arthritis Res Ther. 2005;7(3):R439-444

\title{
Therapiemanagement der Spondyloarthritis
}

Die Spondylorathritis (SpA) ist eine rheumatische Erkrankung, die mit fortschreitender Dauer zu Ankylosierung und erheblichem Funktionsverlust führt. Frühzeitige Diagnose und Therapie sind in der Lage, die Progression der SpA zu verzögern, wobei aufgrund der zahlreichen Komorbiditäten ein fächerübergreifendes Vorgehen indiziert ist.

Die Spondyloarthritis (SpA) ist eine entzündliche rheumatische Erkrankung, die das axiale sowie periphere Skelett betrifft. Laut Kriterien der Assessment of SpondyloArthritis international Society (ASAS) wird zwischen einer peripheren und axialen Form unterschieden wobei die axiale SpA durch den entzündlichen Rückenschmerz und die periphere durch Arthritiden vor allem der unteren Extremitäten charakterisiert sind. Häufig ist die axiale SpA mit anderen Organmanifestationen wie Uveitis, Enthesitis, Daktylitis, Psoriasis oder chronisch entzündlichen Darmerkrankungen assoziiert, die in die ASAS-Kriterien zur Diagnose der axialen SpA integriert sind. [1] „Die neuen ASAS-Kriterien haben dazu geführt, dass die Erkrankung nun leichter und schneller diagnostiziert werden kann", so Priv.-Doz. Dr. Johannes Grisar, KH der Barmherzigen Schwestern, Wien.

\section{Treat to Target}

Axiale SpA ist eine invalidisierende Erkrankung. So sind nach 20 Jahren bis zu $36 \%$ der Patienten mit ankylosierender Spondylitis nicht mehr arbeitsfähig. [2] „Da die Erkrankung in jüngeren Lebensphasen beginnt, ist die Hälfte der Patienten im Alter von 50 Jahren bereits in Frühpension", schildert Priv.-Doz. Dr. Josef Hermann, Klin. Abt. f. Rheumatologie und Immunologie der Univ.-Klinik f. Innere Medizin, Graz. Da SpA eine progrediente Erkrankung ist, sind rasche Diagnose und früher Therapiebeginn wichtig.

Von einer internationalen TaskForce wurden Therapieziele in der Behandlung definiert, wobei die Maximierung der Lebensqualität sowie der sozialen Partizipation im Vordergrund stehen. [3] „Voraussetzung dafür ist, dass Patienten möglichst beschwerdefrei sind, die Funktionalität erhalten bleibt und die Behandlung nicht toxisch ist", sagt Hermann. Einen wesentlichen Beitrag zur Verbesserung der Lebensqualität und sozialen Partizipation scheint die Entzündungshemmung zu leisten. So konnte gezeigt werden, dass Patienten mit ankylosierender Spondylitis, die einen TNF-Blocker erhalten haben, eine deutliche Verbesserung ihrer Lebensqualität erfahren. [4]

Von der Task-Force wurde darüber hinaus festgestellt, dass durch Messung der Krankheitsaktivität und entsprechender Therapieanpassung zum Erreichen niedriger Krankheitsaktivität die langfristige Invalidität und Mortalität vermindert werden können. [3] Das Therapieziel ist die Remission (Fehlen klinischer und laborchemischer Entzündungszeichen), inklusive der extraartikulären Manifestationen. Ein einmal erreichtes Therapieziel sollte aufrecht erhalten werden. So konnte gezeigt werden, dass bei Patienten, die mit einem TNF-Blocker in Remission gelangten, nach Absetzen des Biologikums die Krankheitsaktivität wieder anstieg. [5] „Patienten mit aktiver Spondylarthritis, insbesondere mit axialer, benötigen wahrscheinlich auf Dauer eine antiinflammatorische Therapie" schließt Hermann aus diesen Daten.

\section{An einem Strang ziehen}

Die Diagnose der axialen SpA mit dem Leitsymptom Rückenschmerz - ist eine diagnostische Herausforderung. „Neun von zehn Menschen leiden im Lauf ihres Lebens an Rückenschmerzen, wobei es sich dabei meist um kurze Episoden handelt", schildert Dr. Josef Krugluger, niedergelassener Orthopäde in Purkersdorf (NÖ). Anlaufstellen dieser Patienten sind in erster Linie Notärzte und Hausärzte, aber auch Fachärzte wie Orthopäden oder Neurologen. SpAPatienten rekrutieren sich aus der Gruppe der Patienten mit chronischen Rückenschmerzen, Schmer- zen, die 12 Wochen und länger andauern. Orthopädische Differenzialdiagnosen wie Bandscheibenschäden, Nervenwurzelbeeinträchtigungen oder entzündliche Erkrankungen sind im Gegensatz dazu meist mit akuten bis subakuten Rückenschmerzen assoziiert.

„Zwischen fünf und zehn Prozent der Patienten mit chronischen Rückenschmerzen haben tatsächlich eine axiale SpA, wobei die in Frage kommenden $\mathrm{Pa}$ tienten schwierig zu führen sind", sagt Krugluger. SpA-Patienten sind Menschen im Alter von rund 40 Jahren, die voll im Leben stehen und die möglichst schnell und mit geringem Aufwand eine Diagnose und Therapie erhalten wollen. Dr. Tamas Palotai, Rheumasonderkrankenanstalt Baden der Nö. GKK, erklärt, wie wichtig der Faktor Zeit für die Diagnose ist; Zeit für gründliche Anamnese und klinische Untersuchung, Zeit, um das individuelle Krankheitsbild mit allen Komorbiditäten zu erfassen sowie für Röntgen- und MRT-Untersuchung und HLA-B27-Bestimmung. Aufgrund der zahlreichen möglichen Komorbiditäten bei axialer SpA wie Psoriasis, entzündliche Darmerkrankung oder Uveitis ist bereits in diesem Stadium eine intensive Zusammenarbeit mehrerer Fachrichtungen erforderlich. „Der behandelnde Arzt ist dazu aufgefordert, dem Patienten diese Zusammenhänge aufzuzeigen, wodurch die Motivation für weitergehende diagnostische Schritte steigt", so Krugluger. Eine ausufernde Diagnostik ist laut Krugluger jedoch zu vermeiden. Aber auch Ärzte aller beteiligten Fachdisziplinen wie Augenärzte, Chirurgen, Internisten oder Dermatologen sind angehalten, bei entsprechenden Krankheitsbildern an eine mögliche axiale SpA zu denken, und den Kontakt zur Rheumatologie zu suchen.

Mag. Harald Leitner 